Published in final edited form as:

J Cardiovasc Electrophysiol. 2014 November ; 25(11): 1272-1274. doi:10.1111/jce.12536.

\title{
ICE Imaging of the Left Atrial Appendage
}

\author{
Christopher V. DeSimone, MD, $\mathrm{PhD}^{1}$ and Samuel J. Asirvatham, $\mathbf{M D}^{1,2}$ \\ ${ }^{1}$ Division of Cardiovascular Diseases, Department of Internal Medicine, Mayo Clinic, Rochester, \\ Minnesota \\ ${ }^{2}$ Department of Pediatrics and Adolescent Medicine, Mayo Clinic, Rochester, Minnesota
}

\section{Keywords}

intracardiac echocardiography; ICE; left atrial appendage; ligation; stroke; imaging

Imaging of the left atrial appendage (LAA) is useful for excluding thrombus, facilitating LAA ablation and exclusion procedures, and is an important adjunct for physiological assessment of LAA function. ${ }^{1-3}$

The gold standard for thrombus evaluation in the LAA is transesophageal echocardiography (TEE). ${ }^{1,4,5}$ However, for real-time guidance of invasive procedures that require venous access anyway, intracardiac echocardiography (ICE) has the advantage of easy incorporation into the procedure without the need for esophageal manipulation and general anesthesia. However, obtaining high resolution images of the LAA with ICE is challenging even for experienced operators. ${ }^{4}$ Thus, in contemporary LAA occlusion procedures, despite already available venous access, concurrent TEE is performed.

In this installment of Techniques \& Technology, Fassini et al ${ }^{6}$ explain in clearly elucidated steps, the technique and expected results with ICE imaging from the left atrium (LA) to guide occlusion of the LAA. ${ }^{6}$ The authors outline the essential knowledge of LAA anatomy required for safe and successful occlusion and also describe adjunctive angiography in obtaining supplementary information. ${ }^{6}$

The sole use of ICE in these patients was because of significant patient comorbidities that precluded additional TEE evaluation. For the reader to place in context their described technique for possible, more widespread use of ICE alone for LAA visualization we have provided a review of techniques to potentially optimize LAA images from standard (RA) and less commonly used ICE probe locations; right ventricular outflow tract (RVOT) and left pulmonary artery (LPA). ${ }^{1,7}$ We provide an animation video (Video 1) and ICE images Figure $1 \mathrm{~A}-\mathrm{C}$ ) to help the operator correlate in real time both the probe manipulation and the anatomic views obtained.

Corresponding Author: Samuel J. Asirvatham, MD, Professor of Medicine, Division of Cardiovascular Diseases, 200 First Street SW, Rochester, MN 55905, asirvatham.samuel@ mayo.edu, Tel: 507-293-3376, Fax: 507-255-2550.

Disclosures: S. J. Asirvatham receives no significant honoraria and is a consultant with Abiomed, Atricure, Biosense Webster, Biotronik, Boston Scientific, Medtronic, Spectranetics, St. Jude, Sanofi-Aventis, Wolters Kluwer, Elsevier. C.V. DeSimone has nothing to disclose. 


\section{Imaging from the Right Atrium}

Interventional electrophysiologists routinely place the ICE probe in the right atrium to guide transseptal puncture and various ablation procedures. ${ }^{8}$ Interventional cardiologists are very familiar with inter-atrial septal imaging from the right atrium (RA) used for atrial septal defect and patent foramen ovale closure procedures. ${ }^{9}$ However, LAA imaging from the RA is difficult and highly limited both because of distance from the probe to the LAA and the unique relative regional anatomy of the atria and LAA.

\section{Anatomic considerations}

The LA does not lie directly leftward of the RA but is rather oriented posterior to the RA. Thus, when ICE is used from the RA to guide transseptal puncture, the probe is positioned in the anterior RA and the imaging plane directed posteriorly. Excellent inter-atrial septal images are obtained, and viewing through the septum in the same planar orientation, the LA, and posteriorly located pulmonary veins are typically well-visualized. ${ }^{8}$ However, the LAA is the most anterior portion of the LA and in fact overlies the left ventricle, with the tip of the LAA lying in close proximity to the distal right ventricular outflow tract - the most anterior structure in the heart. This nearly perpendicular orientation to the axis of the LAA to the axis of usual LA imaging from the RA necessarily results in poor imaging quality (Figure 1A) and the inability of the operator to use these images to guide procedures.

\section{Technical considerations}

Attempting to image the LA through the interatrial septum from the RA is also potentially severely limited by the presence of transseptal sheaths, deployed catheters, device delivery systems, and their associated artifacts. The distance from the RA to the LAA can be significant and reach the limits of ICE imaging depths while maintaining reasonable resolution. Although the operator tries to correct for this by bringing the imaging probe close to the septum, the above-mentioned difficult imaging axis relative to the LAA axis and artifact does not allow the expected improvement with trying to get as close as possible to the LAA.

Paradoxically, the best images may be obtained by moving farther back laterally in the RA and attempting to image through the anterior septum (rather than the fossa ovalis). This can be done by deflecting the probe retrograde (laterally) in the low RA near the inferior vena cava or from the crux formed between the superior vena cava, ostium of the right atrial appendage, and tricuspid annulus. A relatively small 3-dimensional window, bounded by the aorta and related shadows more anteriorly and the poor imaging axis of the fossa plane posteriorly, is available and requires some diligence in both obtaining and maintaining the appropriate view through the critical steps of the LAA occlusion procedure. Further limitations include the inability to use higher frequency imaging from such distant locations and relative probe instability as a result of manipulating the primary sheaths used to deploy the occlusion device at the LAA ostium. Despite these efforts to optimize the image, one rarely can obtain a true long axis view of the LAA, and the tip, particularly of an anterior lobe, is generally not possible. 


\section{Imaging from the Coronary Sinus}

The ICE imaging probe can be placed within the coronary sinus (CS) to image the LA, left ventricular and the LAA. ${ }^{7}$

\section{Anatomic considerations}

The CS itself has no direct anatomic relationship with the LAA. ${ }^{10}$ However, the great cardiac vein (GCV) and proximal anterior interventricular vein (AIV) lie in proximity in a complex fashion, sequentially, with various parts of the LAA. The GCV courses close to the left circumflex coronary artery which in turn is related to the inferior and lateral portions of the LAA ostium. As the GCV turns towards the ventricle to become the AIV, then the inferolateral portion of the proximal LAA and its primary lobes now lie in proximity posterior and superior to the course of this vein.

\section{Technical considerations}

Utmost care is required when manipulating a large and relatively stiff probe such as the ICE catheter in the thin-walled tortuous coronary venous system. The authors prefer to never push the probe into the coronary venous system but rather recommend using a soft-tip catheter to cannulate the CS over which a guiding sheath is placed in the GCV/AIV junction. The ICE probe is then placed through the sheath to its tip but not protruding out of the sheath. The sheath is then retracted back over the ICE catheter and then all manipulations described below are done with the ICE probe (and sheath) moving back towards the CS ostium, thus minimizing the risk of perforation.

At the GCV/AIV junction, the probe is rotated so as to point posteriorly and superiorly where cross-sectional and oblique views of the proximal LAA can be obtained. The sheath and probe are then withdrawn into the GCV. Here the probe is gently rotated clockwise from the prior orientation to obtain partial long axis views of the primary LAA lobes and, more posteroinferiorly, the LAA ostium, often visualizing an oblique section, the circumflex coronary artery. By further withdrawing the probe into the GCV and nearing this vein's junction with the CS, the probe is now rotated counterclockwise, directing the imaging plane superiorly with slight retroflection to obtain coronal section images from below upward of the LAA ostium. Then the probe can be rotated 180 degrees clockwise to obtain complementary images of the long axis of the LAA to what was obtained from the GCV/IAV junction. The authors find it valuable to carefully study preprocedural CT or MRI images when available, focusing on the relative anatomy of the coronary venous system and LAA, to anticipate the appropriate manipulation of the probe and likely images obtained from each position.

\section{Imaging from the Right Ventricular Outflow Tract, Main Pulmonary Artery, and Left Pulmonary Artery}

Second only to the RA, the right ventricular outflow tract (RVOT) is a commonly used and familiar location for interventionalists to place the ICE probe, typically used to visualize the pulmonary valve and left ventricular cavity for ablation procedures. The RVOT, main 
pulmonary artery (PA), and left pulmonary artery (LPA) are excellent vantage points to image the LAA once the complex relationship between these structures is appreciated. No single position in the RVOT or pulmonary arterial system, however, allows complete delineation of LAA anatomy.

\section{Anatomic considerations}

The RVOT is the most anterior structure in the normal human heart, while the LAA is the most anterior structure of the LA and in fact lies alongside and to the left of the distal RVOT and PA. The distal PA and LPA then course directly superior and the posterosuperiorly to the LAA roof, separated only by the transverse sinus of the pericardial space. ${ }^{10}$ Inferior branches of the LPA (lingula and lower lobes) are located left and lateral to the LAA. Thus, the advantages of the relative anatomy of the RVOT-LPA axis and the LAA allow for the possibility of obtaining detailed and comprehensive images of the LAA.

\section{Technical considerations}

From a starting position in the mid RA, the ICE probe is deflected anteriorly to enter the RV inflow-outflow junction just distal to the usual position of the bundle of His. Clockwise rotation will sequentially move the imaging plane from a short axis view of the left ventricular inlet at the base to the ostium of the LAA. By gently advancing the probe forward, outstanding views of the LAA ostium, particularly its relationship with the left superior pulmonary vein and the intervening vein of Marshall Ridge can be obtained (Figure 1B). When releasing the deflection that was placed on the probe to enter the RV and maintaining the clockwise torque applied, an oblique or long axis view of the proximal trunk of the LAA, particularly with the windsock-type LAA morphology, can be obtained.

If the probe is now advanced to the PA and the imaging plane rotated inferiorly (usually counterclockwise), then excellent long axis views of the distal LAA primary lobes are seen (Figure 1C). An en face of the LAA - foreshortened view of the tip towards the ostium - can be obtained by pulling back the probe using retroflection from the PA to the RVOT-PA junction. Essentially, one is looking straight at the appendage from this position. Next, the probe can be advanced into the left main PA and potentially into its more inferior subdivision. Long axis views of the LAA, including from the lateral osteal region can provide outstanding detail of the internal architecture of the LAA and its lobular structure.

Fassini et $\mathrm{al}^{6}$ describe their technique for LAA visualization via transseptal placement of the ICE probe in the LAA. Operators should be cognizant of the relative anatomy and techniques to optimize image quality from other right-sided cardiac structures such as the RA, CS, and RVOT-PA axis. These visualization vantage points are by no means exclusive, and complementary images, for example from the PA and LPA medial and superior to the LAA along with those from the GCV and GCV-AIV junction, which is inferior and anterior to the LAA, allow for essentially a complete circle of imaging positions to aid LAA visualization during interventional procedures.

\section{Supplementary Material}

Refer to Web version on PubMed Central for supplementary material. 


\section{Acknowledgments}

The authors wish to thank Dr. Suraj Kapa for help in providing the ICE imaging videos.

\section{Abbreviations}

$\begin{array}{ll}\text { AIV } & \text { anterior interventricular vein } \\ \text { CS } & \text { coronary sinus } \\ \text { GCV } & \text { great cardiac vein } \\ \text { ICE } & \text { intracardiac echocardiography } \\ \text { LA } & \text { left atrium } \\ \text { LAA } & \text { left atrial appendage } \\ \text { LPA } & \text { left pulmonary artery } \\ \text { RA } & \text { right atrium } \\ \text { RVOT } & \text { right ventricular outflow tract } \\ \text { PA } & \text { main pulmonary artery } \\ \text { TEE } & \text { transesophageal echocardiography }\end{array}$

\section{References}

1. Blendea D, Heist EK, Danik SB, Barrett C, Ruskin JN, Mansour M. Analysis of the left atrial appendage morphology by intracardiac echocardiography in patients with atrial fibrillation. $\mathrm{J}$ Interv Card Electrophysiol. 2011; 31:191-196. [PubMed: 21455699]

2. MacDonald ST, Newton JD, Ormerod OJ. Intracardiac echocardiography off piste? Closure of the left atrial appendage using ICE and local anesthesia. Catheter Cardiovasc Interv. 2011; 77:124-127. [PubMed: 20517996]

3. Themistoclakis S, Rossillo A, Bonso A, Raviele A. Intracardiac echocardiography for implantation of LAA occlusion devices: a further step toward the ICE era? Heart Rhythm. 2007; 4:572-574. [PubMed: 17467622]

4. Baran J, Stec S, Pilichowska-Paszkiet E, Zaborska B, Sikora-Frac M, Krynski T, Michalowska I, Lopatka R, Kulakowski P. Intracardiac echocardiography for detection of thrombus in the left atrial appendage: comparison with transesophageal echocardiography in patients undergoing ablation for atrial fibrillation: the Action-Ice I Study. Circ Arrhythm Electrophysiol. 2013; 6:1074-1081. [PubMed: 24243787]

5. Ren JF, Callans DJ. Intracardiac echocardiography with different approaches for imaging of left atrial appendage. Heart Rhythm. 2006; 3:623. author reply 623-624. [PubMed: 16648077]

6. Fassini G, Russo AD, Conti S, Tondo C. An Alternative Transseptal Intracardiac Echocardiography Strategy to Guide Left Atrial Appendage Closure: The First Described Case. J Cardiovasc Electrophysiol. 2014

7. Reddy VY, Neuzil P, Ruskin JN. Intracardiac echocardiographic imaging of the left atrial appendage. Heart Rhythm. 2005; 2:1272-1273. [PubMed: 16253923]

8. Asirvatham SJ, Bruce CJ, Friedman PA. Advances in imaging for cardiac electrophysiology. Coron Artery Dis. 2003; 14:3-13. [PubMed: 12629322]

9. Medford BA, Taggart NW, Cabalka AK, Cetta F, Reeder GS, Hagler DJ, Johnson JN. Intracardiac Echocardiography during Atrial Septal Defect and Patent Foramen Ovale Device Closure in Pediatric and Adolescent Patients. J Am Soc Echocardiogr. 2014 
10. Lachman N, Syed FF, Habib A, Kapa S, Bisco SE, Venkatachalam KL, Asirvatham SJ. Correlative anatomy for the electrophysiologist, Part I: the pericardial space, oblique sinus, transverse sinus. J Cardiovasc Electrophysiol. 2010; 21:1421-1426. [PubMed: 20731740] 

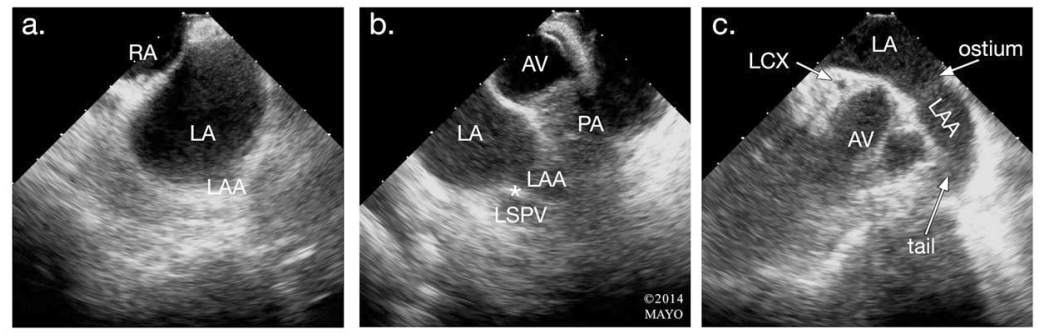

Figure 1. Intracardiac Echocardiographic views of the left atrial appendage

Panel A. With the ICE probe located in the standard right atrial position, there is poor visualization and delineation of the morphology and surrounding structures of the left atrial appendage (LAA). Panel B. With the ICE probe advanced to the right ventricular outflow tract (RVOT), the LAA is well visualized as well as the endocardial ridge or "Q-tip" (asterix) that separates the ostium of the LAA and left superior pulmonary vein (LSPV). Panel C. Further advancement of the ICE probe to the left pulmonary artery provides exceptional visualization of the left atrial appendage. Adequate imaging of the ostium and its relationship to the left circumflex artery (LCX) can be appreciated. The ostial diameter, longitudinal and horizontal axes of the LAA can be seen from this view. RA; right atrium. LA; left atrium. AV; aortic valve. 